\title{
Quiste broncogénico en el período neonatal
}

\author{
Carmen Arreaga S. ${ }^{1}$; Guillermo Correía D. ${ }^{2}$; Katiuska Reynaldos G. ${ }^{3}$
}

\section{Congenital bronchogenic cyst}

Congenitol bronchopulmonary malformations are unicommon cna should be considered in the differential diagnosis of respiratory distress. A prelerm infont is ciescribed who presented at birth with progressively worsening respiraton distress. A bronchogenic cyst of the rain lett broncrus, $3 \mathrm{~cm}$ diameler, vas suspected by computed axial romogropy and comfirmed after surgery. Our patient is so ror dong well even though he shows scme radiological evidence of slight bronchicl stenosis at the site of cyst excision.

[Koy words: bronchogenic cyst.]

Las malformaciones quísticas del árbol traqueobronquial son de origen congénitol. 2 y pueden manifestarse en el periodo de recién nacido o posteriormente y consisten principalmente en cuatro anomalías bien definidas: secuestro puimonar, malformaciones adenomatoídeas quísticas, enfisema lobar congénito y quistes broncogénicos. La localización de los quistes broncogénicos depende del momento del desarollo embriologico en que se produce el trastorno del desarrollo que los causa. Si ocurre al inicio de la gestación, el quiste se ubica en el mediastino. Si se produce mas tarde, se desarrollarán en la periferia (parénquima pulmonar). Los quistes son únicos, uniloculares y esféricos, miden entre 2 y $10 \mathrm{~cm}$ de diámetro, su pared puede contener cartílago, músculo liso, glândulas bronquiales y tejido mucoso y en su interior puede haber aire, moco, líquido seroso o combinaciones de ellos.

\section{Caso clínico}

Varón 36 semanas de edad gestacional, peso adecuado, nació por vía vaginal, parto normal, peso al nacer $2540 \mathrm{~g}$, talla $47 \mathrm{~cm}$, apgar 8 al uninuto y 9 a los 5 min. Embarazo controlado, normal, sin incidentes. Se hospitalizó por difi-

1. Unidad de Neonatología, Hospital del Profesor.

2. Servicio de Cirugia Infantil, Hospital Roberto del Río y del Profesor.

3. Enfermera-Matrona. Unidad de Neonatología, Hospital del Profesor. cultad respiratoria desde las dos horas de vida, requiriendo suplementos de oxígeno. Se descartó proceso infeccioso y enfetmedad de membrana bialina. En la radiografía se registraba desviación del mediastioo por lo que parecía una masa que comprimia la carioa y el bronquio fuente izquierdo (figura I). En la ultrasonografía cardíaca se observaba una masa cilíndrica ubicada cerca de la bifurcación bronquial, sin relación con las arterias pulmonares. En la tomografía axial computadorizad̉a (TAC) se registró una imagen redondeada con aspecto de quiste, de $2 \mathrm{~cm}$ de diámetro, ubicada en el mediastíno posterior que comprimia el bronqujo fuente izquierdo. Además había hiperinsuflación del pulmón izquierdo y desplazamiento del corazón hacia el lado derecho, todo lo cual era compatible con la presencia de un quiste broncogénico del mediastino posterior (figura 2). Mediante esofagograma y mediciones de ácido vanilmandélico en orina se descartaron duplicidad esofágica y neuroblastoma respectivamente.

El paciente fue sonetido a toracotomía, en la que se comprobo una masa quística de $3 \mathrm{~cm}$ de diámetro que desplazaba el eśffago y el bronquio fuente derecho, la que fue extirpada dejando drenaje torácico. En el informe anatomopatológico se describió un quiste cuya pared estaba revestida en parte por epitetio respiratorio rodeado de tejido fibroso y fragmentos de cartílago hialino. Además habia otras cavidades de similar estructura, formulándose el diagnóstico de quiste broneogénico. En el período postoperatorio inmediato el paciente requirió ventilacion mecánica. Posteriormente se complicó con neumotórax derecho que requirió drenaje y quilotórax, por lo cual fue tratado con antibióticos, apoyo nutricional con alimentación parenteral y dieta hipolipidica, reaccionando fayorablemente, sì bien tiene estridor laringeo y lianto dísfónico que han sido interpretados como debidos a laringomalasia por la unidad de otorrinolaringologia. Actualmente está en su casa. en buenas condiciones, y tiene, en una radiografía de tórax de control, signos de estenosis leve del bronquio fuente izquierdo (figura 3 ). sin repercusión clínica. 


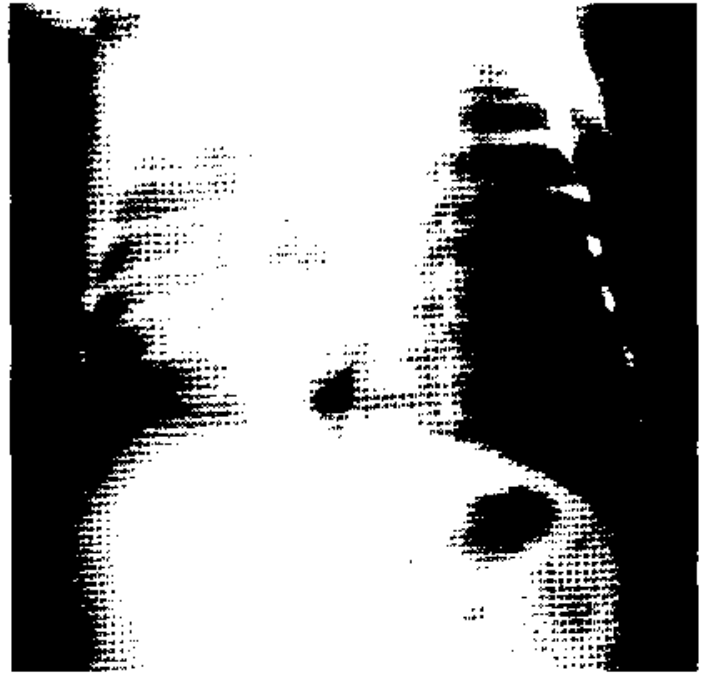

Figura 1: Radiografía de tórax anteroposterior: dusvia ción del mediastino a derceha y compresión de carnia y bronquio fuente izquierdo.

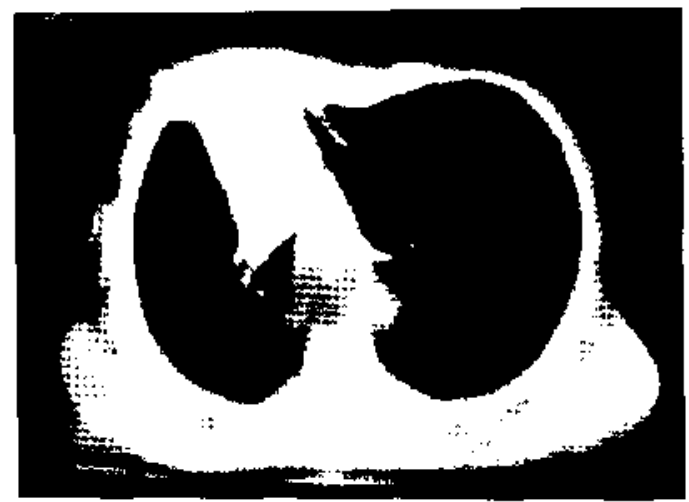

Figura 2: Tomografía axial computadorizada torácica: imagen quistica de $2 \mathrm{~cm}$ en mediastino posterior que comprime bronquio fuente izquierdo.

\section{Comentario}

Los quistes broncogénicos son raros y su diagnóstico en el período neonatal es casi excepcional. Si el diagnóstico se hace precozmente, el pronóstico es bueno. Los quistes broncogénicos son tumores benignos localizados en mediastino posterior o parénquima pulmonar ${ }^{1,2}$ y es posible identificarlos antes del nacimiento por ultrasonografía ${ }^{3}$. Los quistes congénitos pulmonares generalmente son diagnosticados en niños mayores con infecciones respiratorias re- currentes $^{6}$. Cuando se manifiestan en el período neonatal deben ser considerados en el diagnóstico diferencial del sindrome de dificultad respiratoria. En los recién nacidos, los síntomas se dcben a compresión de la tráquea o un bronquio principal y al enfisema lobar secundario ${ }^{7}$ y consisten en estridor, sibilancias y dificultad respiratoria o al alimentarse.

El diagnóstico preoperatorio suele hacerse correctamente en más de tres cuartos de los ca$\operatorname{sos}^{2}$. La radiografía de tórax es útil para identificar las malformaciones congénitas que causan otros casos de sindrome de dificultad respiratoria, pero la tomografía axial ${ }^{4}$ es más eficaz. porque, además de la localización, muestra las características de la lesión.

El tratamiento de elección ${ }^{4}$ en el quiste broncogénico es la resección quirúrgica, especialmente cuando produce síntomas (obstrucción traqueal o bronquial). Ocasionalmente estos quistes pueden infectarse y provocar infecciones pulmonares recurrentes. Las complicaciones postoperatorias que ocurrieron en este paciente fueron esperables. El quilotórax respondió satisfactoriamente al tratamiento médico, como ha ocurrido en otros casos ${ }^{5} \cdot 6$. El barotrauma producido por la ventilación mecánica en el periodo neonatal puediera ser una causa contribuyente al desarrollo de estenosis bronquial en el sitio de la resección del quiste?.

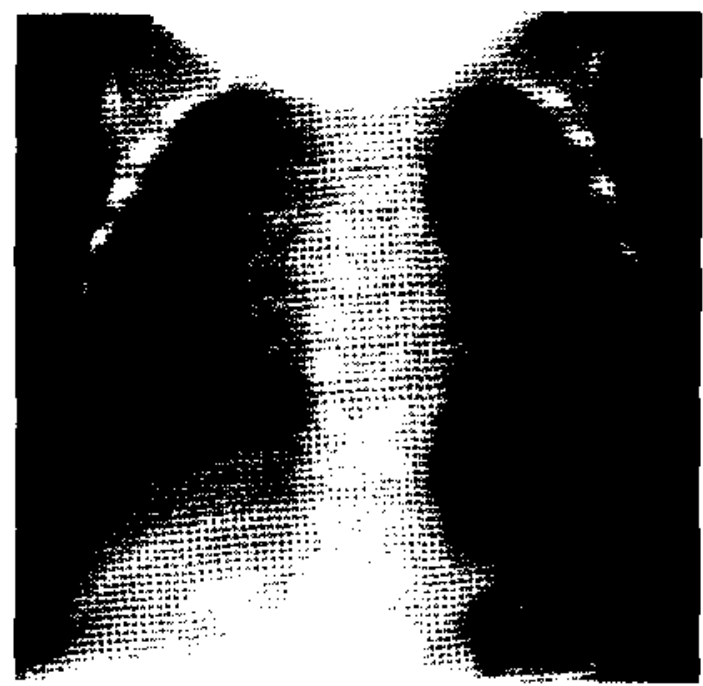

Figura 3: Radiografía de tórax prealta: mediastino centrado, leve estenosis de bronquio fuente izquicrdo. 


\section{Resumen}

Las malformaciones congćnitas del árbol traqueobronquial en el período neonatal son raras y deben ser consideradas en el diagnóstico diferencial del sindrome de dificultad respiratoria del reciến nacido. Se presenta el caso de un reciên nacido pretćrmino con dificultad respiratoria desde el nacimiento, cuyo diagnóstico pre $y$ postoperatorio fuc de un quiste broncogénico de $3 \mathrm{~cm}$ de diámetro que comprimía el bronquio fuente izquierdo. La evolución fuc satisfactoria, quedando una leve cstenosis bronquial en el sitio de la resección del quistc.

(Palabras clave: quiste broncogénico.)

\section{Agradecimientos}

Se agradece a las Dras. Sonia Smith, radióloga infantil. Pátricia Urriola, ccocardiografísta y Eanda Fernández, anatomopal bloga dil Hospital del Profesor.

\section{Referencias}

1. Kraviz R: Congenital malformations of the Iung. Pediatr Clin North Am 1994: 41: 453-472.

2. Dalc $P$. Shaw $N$ : Bronchogenic cyst prescoting in the nconatal period. Acta Puediatr 1994; 83: 110 . 113.

3. Mc Cullagh M, Mac Conmachie I, Garvie D, Dykes E; Accuracy of prenatal diagnosis of congenital cystic adenomatoid malformation. Arch Jis Child 1994: 71: F111-F113.

4. Coran A, Drongmoki $R$ : Congenital cystic disease of the traqueobronquial tree in infants and children. Arch Sury 1994; 129: $521-\$ 27$.

5. Jatene F, Bosisin J, Jutent $M$, es al.: Post traumatic chylothorax. Experience it the postoperative period following cardiothoracic surgery. Arq Bras Cardiol $1993 ; 61: 229-232$.

6. Biley P. Tracy $T$ Ji Cosnors $R$, de Metlo D. Lewis J. Weber $T_{\text {: Congenital Bronchopulmonary malfor- }}$ mations diagnostic and therapeutic considerations. J Thorac Cardiovase \$urg 1990: 99: 597-602.

7. Lazar $R$, Younis $R$, Bissilk $M$ : Btonchogenic cysts: a cause of stridor in the neonate. Am J Otolaryngol 1991; 12: $117-12 \mid$.

\section{AVISO A LOS AUTORES}

Con el objeto de dar prioridad a los trabajos de investigación, en vista de las limitaciones de espacio de la Revista Chilena de Pediatría, el Comité Editorial ha acordado restringir la impresión de casos clínicos a un máximo de dos por cada número. 